\title{
Robust decision making using intuitionistic fuzzy numbers
}

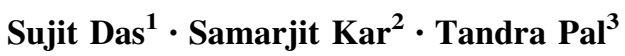

Received: 23 May 2016/Accepted: 23 June 2016/Published online: 5 July 2016

(C) Springer International Publishing Switzerland 2016

\begin{abstract}
Robustness is defined as a system's ability to withstand under disturbances. In real-life applications, where problem parameters are often uncertain, incorporating robustness in decision making is important. In this study, we propose a robust decision making (RDM) approach using intuitionistic trapezoidal fuzzy number (ITrFN). Fuzzy linguistic quantifier (FLQ) is used in the proposed approach to compute the uncertain optimism degree of the decision maker. Initially, decision maker expresses his/her opinion using linguistic terms, which are presented numerically using ITrFNs. The aggregated ITrFN for each of the alternatives is evaluated using intuitionistic trapezoidal fuzzy ordered weighted averaging operator (ITrFOWA). Then, we find out the expected value and variance of the aggregated ITrFN for each alternative, which are subsequently used for robust decision making. A collective measure of these two values of each alternative is considered to find an interval of the corresponding alternative, known as optimal interval. Alternative with maximum optimal interval is selected as the robust solution. Applicability of the proposed approach has been
\end{abstract}

Samarjit Kar

kar_s_k@yahoo.com

Sujit Das

sujit_cse@yahoo.com

Tandra Pal

tandra.pal@gmail.com

1 Department of C.S.E., Dr. B. C. Roy Engineering College, Durgapur, India

2 Department of Mathematics, National Institute of Technology, Durgapur, India

3 Department of C.S.E., National Institute of Technology, Durgapur, India demonstrated on a site selection problem of nuclear power plant. Site selection for installing a nuclear power plant has become a crucial problem throughout the world, especially after the Fukushima (2011) and Chernobyl (1986) nuclear disasters.

Keywords Multi-criteria decision making - Intuitionistic trapezoidal fuzzy number - Intuitionistic trapezoidal fuzzy ordered weighted averaging operator - Robust decision making $\cdot$ Nuclear power plant

\section{Introduction}

A robust system maintains its functionalities under conditions of varying internal or external parameters (Bui et al. 2012). A solution is considered as robust if it is still feasible in the changed scenarios. A robust decision is not necessarily an optimal one. The decision makers need to search for the robust option rather than optimal one, in case of severe uncertainty. When future states are predictable, one may focus on the best or optimal option. However, in case, the future is uncertain, the focus on best option may carry significant risks. Only robust option might be the fruitful choice in these kinds of situations. Robustness is considered as a broader concept of adaptation. Adaptation allows inclusion and deletion of functionalities, whereas robustness tries to self-organize the system through structural changes to maintain the system's functionalities (Bui et al. 2012). Mens et al. (2011) distinguished between system robustness and decision robustness. According to them, system robustness is common in the field of engineering and biology. It refers to the ability of systems to maintain the desired system characteristics when subjected to disturbances. The authors (Mens et al. 2011) defined 
decision robustness as a characteristic of decisions policy, which is used as a criterion for making decisions under uncertainty. They (Mens et al. 2011) stated that a decision or policy is considered to be robust when it performs well under a range of conditions. In this era of uncertainty, when almost everything is uncertain, survival of optimal decision has been facing a bottleneck. Therefore, the need of robust decision is vital in uncertain environment. As uncertainties are well expressed using fuzzy systems, robust decision making (RDM) using fuzzy systems would be a prominent research direction. This motivated us to consider RDM using intuitionistic fuzzy set (IFS), where IFS is considered as the generalizations of fuzzy set. The application of IFS instead of fuzzy set implies the introduction of another degree of freedom into a set description. Here, in addition to membership value, we also have non-membership value and hesitation margin. Being a generalization of fuzzy sets, IFSs give us an additional possibility to represent imperfect knowledge by which it is possible to describe many real problems in a more adequate way.

Robustness in decision making strategy is the flexibility to maintain different possible situations. In real-life applications, where problem parameters are often uncertain, incorporating robustness in decision making is important. Uncertainties are well expressed using qualitative terms rather than quantitative. Fuzzy system has been proved to be effective to deal with uncertainties, where linguistic variables are used to represent the qualitative terms. Due to the existence of fuzziness in human reasoning and real-life decision making problems, it is more reasonable and natural to utilize linguistic information to express decision makers' opinion. The use of linguistic information is inevitable in many real-life situations, where one common approach to model the linguistic information is the fuzzy linguistic approach (Zadeh 1975a, b) that uses the fuzzy set theory to manage the uncertainties. In Zadeh (1975a, b), Zadeh introduced the concept of linguistic variable as "a variable whose values are not numbers but words or sentences in a natural or artificial language". A linguistic value is less precise than a number, but it is more close to human cognitive processes and used successfully to solve problems dealing with uncertainty.

In the literature, robustness has been defined by many researchers in different ways. Gupta and Rosenhead (1968) first applied robustness to decision problems in the context of sequential investment planning, which deals with finding the location of sites for new factories in an industrial expansion program. Rosenhead et al. (1972) further illustrated the concept of robustness, described in Gupta and Rosenhead (1968). According to them (Rosenhead et al. 1972), robustness of a decision is based on the flexibility that it maintains. In Deb and Gupta (2006), Gaspar-Cunha and Covas (2008), and Xue et al. (2007), the authors introduced robustness in multi-objective optimization. Deb and Gupta (2006) provided two definitions of robustness considering uncertain optimization parameters. GasparCunha and Covas (2008) defined different fitness functions for multi-objective evolutionary algorithms that led to robust solutions. Xue et al. (2007) studied robustness with respect to changes in external parameters, where changes are assumed to be stochastic. Decision problems are often subject to uncertainties. In Simon (1959), Simon defined that a robust system performs satisfactorily, i.e., it satisfies the performance criteria over a wide range of uncertain features. According to Lempert and Collins (2007), robust decision making (RDM) is a planning framework designed to help decision makers to formulate plans for the future under the conditions of uncertainty. The authors in Lempert and Collins (2007) also stated that robust decision making (RDM) represents the uncertainty by considering system performance under a wide range of situations. RDM considers the concept of robustness over optimal situation and assumes that a robust strategy is able to satisfy minimum performance criteria over a wide range of possible futures. Studies of robustness in scheduling problem can be found in Yanez and Ramirez (2003), Hasuike (2013), and Dey et al. (2015). Nag et al. (2014) defined robust consensus, and based on it, they provided a mechanism for multiobjective optimization problems. The authors proved that the experimental results are capable of finding solutions having robust consensus in the soft region, specified by the users. In Zarghami et al. (2008), authors introduced robustness in fuzzy multi-criteria decision making (MCDM) using expected value and variance. Zarghami et al. (2008) proposed a new approach known as fuzzy stochastic order weighted averaging (FSOWA) and applied it in watershed management problem to illustrate the robust behaviour of the concerned projects. The authors considered 13 water resource projects under construction in Sefidrud watershed, located in the northwestern region of Iran and evaluated the robustness of the projects based on a set of predefined criteria. Zarghami and Szidarovszky (2009) proposed stochastic fuzzy multi-criteria decision making (MCDM) for robust water resources management. They illustrated their method using a water resources management problem in the central Tisza river in Hungary. The authors also compared their method with some of the existing methods and proved that their method was suitable under uncertainty.

Recently, researchers have concentrated on information granulation for real-life decision making under uncertainties. Human beings often consider information granules because of their inherent imprecise reasoning process. Granules act as the pillars of granular computing ( $\mathrm{GrC})$, which are composed of objects that are combined together by indiscernibility relationship. Granules are considered 
when a problem involves incomplete, uncertain, and vague information, and it is difficult to discern distinct objects. The systems based on granular computing exploit the tolerance for imprecision, uncertainty, approximate reasoning as well as partial truth of soft computing framework, and are capable of achieving tractability, robustness, and close resemblance with human-like (natural) decision making (Bargiela and Pedrycz 2012; Cabrerizo et al. 2013). Robustness in decision making can be achieved by granular computing, since GrC results in higher efficiency and lower energy consumption. Nowadays, GrC has become significant in the design and implementation of robust intelligent systems to solve various real-life applications (Pedrycz and Chen 2015; Peters and Weber 2016; Livi and Sadeghian 2016; Xu and Wang 2016; Antonelli et al. 2016; Mendel 2016; Lingras et al. 2016; Skowron et al. 2016; Dubois and Prade 2016; Loia et al. 2016; Yao 2016; Ciucci 2016; Kreinovich 2016; Wilke and Portmann 2016; Min and Xu 2016; Maciel et al. 2016; Apolloni et al. 2016; Song and Wang 2016; Liu et al. 2016).

Since the introduction of intuitionistic fuzzy set (IFS) (Atanassov 1986, 1999), researchers have successfully applied it in decision making problems. IFS is a generalized version of fuzzy set and well suited to deal with uncertainty. In this paper, we propose a robust multi-criteria decision making (MCDM) approach using intuitionistic trapezoidal fuzzy numbers (ITrFNs). Due to the complex and vague nature of human judgement, decision makers often prefer to express their opinions using linguistic terms instead of exact numerical assessments. In this study, initially, decision maker provides his/her opinion using linguistic terms, which are presented using ITrFNs. Intuitionistic trapezoidal fuzzy ordered weighted averaging (ITrFOWA) operator is used to aggregate the ITrFNs for alternatives corresponding to various criteria. Score and accuracy values of those aggregated ITrFNs are computed to find the optimal solution. As we know that optimal solution may not be a robust one, both expected values and variances of the aggregated ITrFNs for each of the alternatives are determined. Finally, these two measures are combined to evaluate a collective measure and used to find an interval, known as optimal interval for each alternative. Robust decision is made by selecting the alternative with maximum optimal interval.

Considering the various risks associated with nuclear power plant, the proposed method has been applied to find a favorable location for installing nuclear power plant. Nuclear accidents in Fukushima (2011) and Chernobyl (1986) have raised an intensive awareness and concern regarding the expansion of nuclear energy portfolio, which may be socially too risky relative to its benefits compared to other alternative resources. The issue of risk benefit trade-off with respect to nuclear power is not a new issue, but the trade-off margin that was socially acceptable prior to Fukushima exists no more. This has led to re-evaluate the role of nuclear power in future energy plan in many countries. Public protests against nuclear power have widened and become more intense (Srinivasan and Rethinaraj 2013). According to the pioneer of nuclear reactor, Weinberg (1986), "nuclear accident anywhere is a nuclear accident everywhere".

To study the applicability of robustness in MCDM, the rest of the article is organized as follows. Section 2 presents the basic ideas relevant to this study. Section 3 extends ITrFOWA operator using optimism degree, FLQ, and RIM quantifier. The proposed algorithmic approach for robust decision making is also presented here. An application of the proposed algorithm in nuclear site selection problem is demonstrated in Sect. 4 followed by result discussion and comparison in Sect. 5. Finally, conclusions are drawn in Sect. 6.

\section{Preliminaries}

In this section, we recall some basic concepts of ITrFNs and ITrFOWA operator. We also present some operations on ITrFNs. In intuitionistic trapezoidal fuzzy set (ITrFS) (Wang and Zhang 2009b), the membership and nonmembership functions are expressed by trapezoidal fuzzy numbers. ITrFS is considered to be more powerful in expressing the uncertainty than intuitionistic fuzzy set. The basis of ITrFS is intuitionistic trapezoidal fuzzy number (ITrFN). Wang and Zhang (2009b) defined the concept of intuitionistic trapezoidal fuzzy numbers and their operational laws (Xu and Yager 2006).

Definition 2.1 (Intuitionistic trapezoidal fuzzy number) Let $a$ be an ITrFN. Its membership function is defined as:

$\mu_{\dot{a}}(x)=\left\{\begin{array}{cc}\frac{x-a}{b-a} \mu_{\dot{a}}, & a \leq x<b ; \\ \mu_{a}, & b \leq x \leq c ; \\ \frac{d-x}{d-c} \mu_{\dot{a}}, & c<x \leq d ; \\ 0, & \text { otherwise. }\end{array}\right.$

And its non-membership function is defined as:

$$
v_{\dot{a}}(x)=\left\{\begin{array}{cc}
\frac{b-x+v_{\dot{a}}\left(x-a_{1}\right)}{b-a_{1}} \mu_{\dot{a}}, & a_{1} \leq x<b ; \\
v_{a}, & b \leq x \leq c ; \\
\frac{x-c+v_{\dot{a}}\left(d_{1}-x\right)}{d_{1}-c} \mu_{\dot{a}}, & c<x \leq d_{1} ; \\
0, & \text { otherwise }
\end{array}\right.
$$

Here, $\quad 0 \leq \mu_{\dot{a}} \leq 1,0 \leq v_{\dot{a}} \leq 1, \quad$ and $\quad \mu_{\dot{a}}+v_{\dot{a}} \leq 1, \quad a, b, c$, $d, a_{1}, d_{1} \in \mathfrak{R} . \dot{a}=\left\langle\left([a, b, c, d]: \mu_{\dot{a}}\right),\left(\left[a_{1}, b, c, d_{1}\right]: v_{\dot{a}}\right)>\right.$ 
called an $\operatorname{ITrFN}$. For convenience, $\operatorname{ITrFN} a ́$ is written as $\dot{a}=\left([a, b, c, d] ; \mu_{\dot{a}}, v_{\dot{a}}\right)$.

Let $\dot{\alpha}_{1}=\left(\left[a_{1}, b_{1}, c_{1}, d_{1}\right] ; \mu_{\dot{\alpha}_{1}}, v_{\dot{\alpha}_{1}}\right)$ and $\dot{\alpha}_{2}=\left(\left[a_{2}, b_{2}, c_{2}, d_{2}\right]\right.$; $\left.\mu_{\dot{\alpha}_{2}}, v_{\dot{\alpha}_{2}}\right)$ be two ITrFNs, and $\lambda \geq 0$, then following operations are defined on $\dot{\alpha}_{1}$ and $\dot{\alpha}_{2}$.
1. $\quad \dot{\alpha}_{1} \oplus \dot{\alpha}_{2}=\left(\left[a_{1}+a_{2}, b_{1}+b_{2}, c_{1}+c_{2}, d_{1}+d_{2}\right] ; \mu_{\dot{\alpha}_{1}}+\right.$ $\left.\mu_{\dot{\alpha}_{2}}-\mu_{\dot{\alpha}_{1}} \mu_{\dot{\alpha}_{2}}, v_{\dot{\alpha}_{1}} v_{\dot{\alpha}_{2}}\right)$

2. $\quad \dot{\alpha}_{1} \otimes \dot{\alpha}_{2}=\left(\left[a_{1} a_{2}, b_{1} b_{2}, c_{1} c_{2}, d_{1} d_{2}\right] ; \mu_{\dot{\alpha}_{1}} \mu_{\dot{\alpha}_{2}}, v_{\dot{\alpha}_{1}}+v_{\dot{\alpha}_{2}}\right.$ $\left.-v_{\alpha_{1}} v_{\dot{\alpha}_{2}}\right)$

3. $\lambda \dot{\alpha}_{1}=\left([\lambda a, \lambda b, \lambda c, \lambda d] ; 1-\left(1-\mu_{\dot{\alpha}_{1}}\right)^{\lambda}, v_{\dot{\alpha}_{1}}^{\lambda}\right)$

4. $\quad \dot{\alpha}_{1}^{\lambda}=\left(\left[a^{\lambda}, b^{\lambda}, c^{\lambda}, d^{\lambda}\right] ; \mu_{\dot{\alpha}_{1}}^{\lambda}, 1-\left(1-v_{\dot{\alpha}_{1}}\right)^{\lambda}\right)$

Definition 2.2 (Intuitionistic trapezoidal fuzzy decision matrix) The intuitionistic trapezoidal fuzzy decision matrix (Wei 2010) is defined as $\dot{R}=(\dot{r})_{m \times n}=$ $\left(\left[a_{i j}, b_{i j}, c_{i j}, d_{i j}\right] ; \mu_{i j}, v_{i j}\right)_{m \times n}$, where $m$ and $n$ are, respectively, the number of alternatives and number of criteria, and $i=1,2, \ldots, m, j=1,2, \ldots, n$. Here, $\quad 0 \leq a_{i j} \leq b_{i j}$ $\leq c_{i j} \leq d_{i j} \leq 1,0 \leq \mu_{i j}, v_{i j} \leq 1,0 \leq \mu_{i j}+v_{i j} \leq 1$.

Intuitionistic trapezoidal fuzzy positive ideal solution and intuitionistic trapezoidal fuzzy negative ideal solution

The intuitionistic trapezoidal fuzzy positive ideal solution $r^{+}$and intuitionistic trapezoidal fuzzy negative ideal solution $\dot{r}^{-}$are defined as follows:

$\dot{r}^{+}=\left(\left[a^{+}, b^{+}, c^{+}, d^{+}\right] ; \mu^{+}, v^{+}\right)=([1,1,1,1] ; 1,0)$

$\dot{r}^{-}=\left(\left[a^{-}, b^{-}, c^{-}, d^{-}\right] ; \mu^{-}, v^{-}\right)=([0,0,0,0] ; 1,0)$

Let $\dot{\alpha}_{i}=\left(\left[a_{i}, b_{i}, c_{i}, d_{i}\right] ; \mu_{\dot{\alpha}_{i}}, v_{\dot{\alpha}_{i}}\right)$ be an intuitionistic trapezoidal fuzzy number and $\dot{r}^{+}$be an intuitionistic trapezoidal fuzzy positive ideal solution, then the distance between $\dot{\alpha}_{i}$ and $\dot{r}^{+}$is denoted as $d\left(\dot{\alpha}_{i}, \dot{r}^{+}\right)$. If $d\left(\dot{\alpha}_{1}, \dot{r}^{+}\right)<d\left(\dot{\alpha}_{2}, \dot{r}^{+}\right)$, then $\dot{\alpha}_{1}>\dot{\alpha}_{2}$.

\subsection{Score function and accuracy function}

To compare any two intuitionistic trapezoidal fuzzy numbers, Wang and Zhang (2009a) defined the score function, accuracy function, and expected value as given below.
Definition 2.3 (Score and accuracy function of ITrFN) The score function $S(\dot{a})$ and accuracy function $H(\dot{a})$ of the $\operatorname{ITrFN} \dot{a}=\left([a, b, c, d] ; \mu_{\dot{a}}, v_{a}\right)$ are respectively defined as

$$
S(\dot{a})=I(\dot{a}) \times\left(\mu_{\dot{a}}-v_{\dot{a}}\right)
$$

and

$$
H(\dot{a})=I(\dot{a}) \times\left(\mu_{\dot{a}}+v_{a}\right),
$$

where

$$
I(\hat{a})=\frac{1}{8} \times\left[(a+b+c+d) \times\left(1+\mu_{\dot{a}}-v_{a}\right)\right]
$$

is the expected value of the $\operatorname{ITrFN} a$.

Using the score and accuracy functions, two ITrFNs $\dot{\alpha}_{1}=\left(\left[a_{1}, b_{1}, c_{1}, d_{1}\right] ; \mu_{\dot{\alpha}_{1}}, v_{\dot{\alpha}_{1}}\right) \quad$ and $\quad \dot{\alpha}_{2}=\left(\left[a_{2}, b_{2}, c_{2}, d_{2}\right]\right.$; $\left.\mu_{\dot{\alpha}_{2}}, v_{\alpha_{2}}\right)$ can be compared as follows:

1. If $S\left(\dot{\alpha}_{1}\right)<S\left(\dot{\alpha}_{2}\right)$, then $\dot{\alpha}_{1}$ is smaller than $\dot{\alpha}_{2}$, i.e., $\dot{\alpha}_{1}<\dot{\alpha}_{2}$.

2. If $S\left(\dot{\alpha}_{1}\right)=S\left(\dot{\alpha}_{2}\right)$, then

(a) If $H\left(\dot{\alpha}_{1}\right)<H\left(\dot{\alpha}_{2}\right)$, then $\dot{\alpha}_{1}$ is smaller than $\dot{\alpha}_{2}$, i.e., $\dot{\alpha}_{1}<\dot{\alpha}_{2}$

(b) If $H\left(\dot{\alpha}_{1}\right)=H\left(\dot{\alpha}_{2}\right)$, then $\dot{\alpha}_{1}$ and $\dot{\alpha}_{2}$ represent the same information, i.e., $\alpha_{1}=\dot{\alpha}_{2}$.

(c) If $H\left(\dot{\alpha}_{1}\right)>H\left(\dot{\alpha}_{2}\right)$, then $\dot{\alpha}_{1}$ is larger than $\dot{\alpha}_{2}$, i.e., $\dot{\alpha}_{1}>\dot{\alpha}_{2}$.

The variance of ITrFN (Wang and Tian 2010) provides a measure of the spread of the distribution of ITrFNs around its expected value. A small value of variance indicates that the ITrFN is firmly concentrated around its expected value, and a large value of variance indicates that the ITrFN has a wide spread around the expected value.

Definition 2.4 (Variance of $I T r F N$ ) The variance $V(a)$ of the $\operatorname{ITrFN} \dot{a}^{\prime}=\left([a, b, c, d] ; \mu_{\dot{a}}, v_{\dot{a}}\right)$ is defined as follows:

(i) when $\bar{\alpha}=\bar{\beta}, v(a ́)=\frac{3(b-a+\bar{\alpha})^{2}+\bar{\alpha}^{2}}{24}$;

(ii) when $\bar{\alpha}>\bar{\beta}$,

$$
V(\dot{a})=\left\{\begin{array}{cc}
\frac{1}{6}\left[-\frac{(b-m)^{3}}{\bar{\beta}}-\frac{(a-\bar{\alpha}-m)^{3}}{\bar{\alpha}}+\frac{\left.(b \bar{\alpha}+a \bar{\beta}-m(\bar{\alpha}+\bar{\beta}))^{3}\right],}{\bar{\alpha} \bar{\beta}(\bar{\alpha}-\bar{\beta})^{2}}\right] & a-m<0 \\
\frac{1}{6}\left[\frac{(a-m)^{3}}{\bar{\alpha}}-\frac{(b-m)^{3}}{\bar{\beta}}-\frac{(a-\bar{\alpha}-m)^{3}}{\bar{\alpha}}+\frac{(b \bar{\alpha}+a \bar{\beta}-m(\bar{\alpha}+\bar{\beta}))^{3}}{\bar{\alpha} \bar{\beta}(\bar{\alpha}-\bar{\beta})^{2}}\right], & a-m \geq 0
\end{array}\right.
$$


iii) when $\bar{\alpha}<\bar{\beta}$,

$V(\dot{a})=\left\{\begin{array}{cc}\frac{1}{6}\left[\frac{(a-m)^{3}}{\bar{\alpha}}+\frac{(b+\bar{\beta}-m)^{3}}{\bar{\beta}}-\frac{(b \bar{\alpha}+a \bar{\beta}-m(\bar{\alpha}+\bar{\beta}))^{3}}{\bar{\alpha} \bar{\beta}(\bar{\alpha}-\bar{\beta})^{2}}\right], & b-m<0 \\ \frac{1}{6}\left[\frac{(a-m)^{3}}{\bar{\alpha}}-\frac{(b-m)^{3}}{\bar{\beta}}+\frac{(b+\bar{\beta}-m)^{3}}{\bar{\beta}}+\frac{(b \bar{\alpha}+a \bar{\beta}-m(\bar{\alpha}+\bar{\beta}))^{3}}{\bar{\alpha} \bar{\beta}(\bar{\alpha}-\bar{\beta})^{2}}\right], & b-m \geq 0\end{array}\right.$

Here, $\bar{\alpha}=b-a, \bar{\beta}=d-c$, and $m=I(\dot{a})$.

\subsection{Distance measures between ITrFNs}

Wang and Zhang (2009b) and Ye (2012) defined two distance measurement techniques between two intuitionistic trapezoidal fuzzy numbers which are given below.

\subsubsection{Normalized Hamming distance}

The normalized Hamming distance between two ITrFNs $\dot{\alpha}_{1}=\left(\left[a_{1}, b_{1}, c_{1}, d_{1}\right] ; \mu_{\dot{\alpha}_{1}}, v_{\dot{\alpha}_{1}}\right) \quad$ and $\quad \dot{\alpha}_{2}=\left(\left[a_{2}, b_{2}, c_{2}, d_{2}\right]\right.$; $\left.\mu_{\dot{\alpha}_{2}}, v_{\dot{\alpha}_{2}}\right)$ is defined as:

$l_{\text {ITrFN }}\left(\dot{\alpha}_{1}, \dot{\alpha}_{2}\right)=\frac{1}{8}\left(\begin{array}{c}\left|\left(1+\mu_{\dot{\alpha}_{1}}-v_{\dot{\alpha}_{1}}\right) a_{1}-\left(1+\mu_{\dot{\alpha}_{2}}-v_{\dot{\alpha}_{2}}\right) a_{2}\right| \\ +\left|\left(1+\mu_{\dot{\alpha}_{1}}-v_{\dot{\alpha}_{1}}\right) b_{1}-\left(1+\mu_{\dot{\alpha}_{2}}-v_{\dot{\alpha}_{2}}\right) b_{2}\right| \\ +\left|\left(1+\mu_{\dot{\alpha}_{1}}-v_{\dot{\alpha}_{1}}\right) c_{1}-\left(1+\mu_{\dot{\alpha}_{2}}-v_{\dot{\alpha}_{2}}\right) c_{2}\right| \\ +\left|\left(1+\mu_{\dot{\alpha}_{1}}-v_{\dot{\alpha}_{1}}\right) d_{1}-\left(1+\mu_{\dot{\alpha}_{2}}-v_{\dot{\alpha}_{2}}\right) d_{2}\right|\end{array}\right)$

\subsubsection{Normalized Euclidean distance}

The normalized Euclidean distance between two ITrFNs $\dot{\alpha}_{1}=\left(\left[a_{1}, b_{1}, c_{1}, d_{1}\right] ; \mu_{\dot{\alpha}_{1}}, v_{\dot{\alpha}_{1}}\right) \quad$ and $\quad \dot{\alpha}_{2}=\left(\left[a_{2}, b_{2}, c_{2}, d_{2}\right]\right.$; $\left.\mu_{\dot{\alpha}_{2}}, v_{\dot{\alpha}_{2}}\right)$ is defined as:

$e_{\text {ITrFN }}\left(\dot{\alpha}_{1}, \dot{\alpha}_{2}\right)=\sqrt{\frac{1}{8}\left(\begin{array}{c}\left\{\left(1+\mu_{\dot{\alpha}_{1}}-v_{\dot{\alpha}_{1}}\right) a_{1}-\left(1+\mu_{\dot{\alpha}_{2}}-v_{\dot{\alpha}_{\alpha_{2}}}\right) a_{2}\right\}^{2} \\ +\left\{\left(1+\mu_{\dot{\alpha}_{1}}-v_{\dot{\alpha}_{1}}\right) b_{1}-\left(1+\mu_{\dot{\alpha}_{2}}-v_{\dot{\alpha}_{2}}\right) b_{2}\right\}^{2} \\ +\left\{\left(1+\mu_{\dot{\alpha}_{1}}-v_{\dot{\alpha}_{1}}\right) c_{1}-\left(1+\mu_{\dot{\alpha}_{2}}-v_{\dot{\alpha}_{2}}\right) c_{2}\right\}^{2} \\ +\left\{\left(1+\mu_{\dot{\alpha}_{1}}-v_{\dot{\alpha}_{1}}\right) d_{1}-\left(1+\mu_{\dot{\alpha}_{2}}-v_{\dot{\alpha}_{2}}\right) d_{2}\right\}^{2}\end{array}\right)}$

\subsection{Intuitionistic trapezoidal fuzzy ordered weighted averaging operator}

Let $Q$ be the set of ITrFNs and $a_{j}(j=1,2, \ldots, n)$ be a collection of $n$ ITrFNs. An intuitionistic trapezoidal fuzzy ordered weighted averaging (ITrFOWA) operator (Wei 2010; $\mathrm{Xu} 2007$ ) of dimension $n$ is a mapping ITrFOWA:
$Q^{n} \rightarrow Q$, that has an associated vector $\omega=$ $\left(\omega_{1}, \omega_{2}, \ldots, \omega_{n}\right)^{T}$, such that $\omega_{i} \geq 0$ and $\sum_{j=1}^{n} \omega_{j}=1$.

Moreover,

$$
\begin{aligned}
& \operatorname{ITrFOWA}_{\omega}\left(\dot{a}_{1}, \dot{a}_{2}, \ldots, \dot{a}_{n}\right)=\sum_{j=1}^{n} \dot{a}_{\sigma(j)} \omega_{j} \\
& =\left(\left[\sum_{j=1}^{n} \dot{a}_{\sigma(j)} \omega_{j}, \sum_{j=1}^{n} \dot{b}_{\sigma(j)} \omega_{j}, \sum_{j=1}^{n} \dot{c}_{\sigma(j)} \omega_{j}, \sum_{j=1}^{n} \dot{d}_{\sigma(j)} \omega_{j}\right] ; 1\right. \\
& \left.\quad-\prod_{j=1}^{n}\left(1-\mu_{\dot{a}_{\sigma(j)}}\right)^{\omega_{j}}, \prod_{j=1}^{n}\left(v_{a_{\sigma(j)}}\right)^{\omega_{j}}\right),
\end{aligned}
$$

where $(\sigma(1), \sigma(2), \ldots, \sigma(n))$ is a permutation of $(1,2, \ldots, n)$, such that $\dot{a}_{\sigma(j-1)} \geq \dot{a}_{\sigma(j)}$, for any $j$.

\section{Robust decision making using intuitionistic trapezoidal fuzzy number}

In this section, we extend the method given in Zarghami et al. (2008) and Zarghami and Szidarovszky (2009) in the framework of ITrFNs and propose an algorithmic approach to solve MCDM problem using intuitionistic trapezoidal fuzzy ordered weighted averaging (ITrFOWA) operator. This study also extends the ITrFOWA operator using optimism degree of decision maker, fuzzy linguistic quantifier (FLQ), and regular increasing monotonic (RIM) quantifier to find the robust decision.

\subsection{ITrFOWA using optimism degree, FLQ, and RIM quantifier}

Yager (1988) introduced ordered weighted averaging (OWA) operator which has different varieties for different orders of weights. Order weights depend on the optimism degree, also known as orness degree of the decision maker (Yager 2002). Higher the values of the weights at the beginning of the weight vector, more will be the optimism degree. The measure of optimism degree of a decision maker is defined in the interval $[0,1]$. When the optimism 
degree is 1 , the decision maker is considered to be riskprone, and when it is 0 , the decision maker is said to be risk-aversion type. When the optimism degree is more, a decision maker is said to be optimistic. Pessimistic decision maker is assigned less optimism degree. Generally, optimistic decision maker prefers less criteria, while pessimistic decision maker prefers more criteria in their decision making process (Zarghami et al. 2008; Zarghami and Szidarovszky 2009). Normally, an expert/decision maker considers more criteria when he/she prominently avoids the risk of making improper decisions, which may result in conventional decision. But when the decision maker prefers fewer criteria with the hope of finding better decision, then the results will differ from that of the pessimistic decision maker and may be improved.

Definition 3.1 (Optimism degree) Optimism degree (Yager 1988) $\theta$ of a decision maker is defined as:

$\theta=\frac{1}{n-1} \sum_{j=1}^{n}(n-j) w_{j}$

Here, $n$ is the number of attributes/criteria, $w=$ $\left(w_{1}, w_{2}, \ldots, w_{n}\right)^{T}$ is a weight vector associated with those attributes, such that $w_{j} \geq 0$ and $\sum_{j=1}^{n} w_{j}=1$. Different decision makers may consider different weight vectors for the same set of attributes.

FLQ is used to characterize the aggregation process, and in this study, we consider it with RIM quantifier. Some examples of FLQ are: all, half, few or at least one of them. Malczewski (2006) has defined seven RIM quantifiers to aggregate the inputs, which are shown in Table 1. RIM quantifiers are used to model the linguistic inputs, which satisfy the following conditions.

$Q(0)=0, Q(1)=1, \quad$ and $\quad Q\left(p_{1}\right) \geq Q\left(p_{2}\right)$ if $p_{1} \geq p_{2}$

Here, $Q$ is a fuzzy membership function. When RIM quantifier is associated with an OWA operator, the weight vector (Yager 1988, 1996) is obtained as given below in (12).
$w_{j}=Q\left(\frac{j}{n}\right)-Q\left(\frac{j-1}{n}\right), \quad j=1,2, \ldots, n$

Among different possible forms of function $Q$, we have considered $Q(p)=p^{\alpha}$, where $\alpha$ is a positive parameter. Using (11) and (12), the optimism degree can be expressed as follows:

$\theta=\int_{0}^{1} Q(p) \mathrm{d} p=\int_{0}^{1} p^{\alpha} \mathrm{d} p=\frac{1}{(1+\alpha)}, \alpha=\left(\frac{1}{\theta}-1\right)$

Using (12) and (13), (10) can be further expressed as follows in (14).

$$
\begin{aligned}
\operatorname{ITrFOWA}_{\omega}\left(\dot{a}_{1}, \dot{a}_{2}, \ldots, \hat{a}_{n}\right) & =\sum_{j=1}^{n} \dot{a}_{\sigma(j)} \omega_{j} \\
& =\sum_{j=1}^{n} \dot{a}_{\sigma(j)}\left[\left(\frac{j}{n}\right)^{\frac{1}{\theta}-1}-\left(\frac{j-1}{n}\right)^{\frac{1}{\theta}-1}\right]
\end{aligned}
$$

\subsection{Collective measure using expectation and variance}

Decision making in uncertain environment always yields a risk for the decision makers due to the uncertain nature of the decision parameters. When the decision maker does not consider the risk in the decision making process, then his/ her objective is only to get the optimal solution, i.e., to maximize the expected value. But when the decision maker considers the associated risk arising from uncertainty, then he/she attempts also to minimize the variance. More the uncertainty, more is the variance. Expectation and variance are two conflicting objectives. A combination of them, known as collective measure $F_{k}^{*}$ for the $k_{t h}$ alternative is shown in (15) and used to solve the problem.

$F_{k}^{*}=E\left(F_{k}\right)-\xi_{k} \operatorname{var}\left(F_{k}\right), \quad \xi_{k} \geq 0$
Table 1 Family of RIM quantifiers

\begin{tabular}{llll}
\hline Fuzzy linguistic quantifier & Parameter of quantifier $(\alpha)$ & Optimism degree $(\theta)$ & Optimistic condition \\
\hline Atleast one of them & $\alpha \rightarrow 0.0$ & 0.999 & Very optimistic \\
Few of them & 0.1 & 0.909 & Optimistic \\
Some of them & 0.5 & 0.667 & Fairly optimistic \\
Half of them & 1.0 & 0.5 & Neutral \\
Many of them & 2.0 & 0.333 & Fairly pessimistic \\
Most of them & 10.0 & 0.091 & Pessimistic \\
All of them & $\alpha \rightarrow \infty$ & 0.001 & Very pessimistic \\
\hline
\end{tabular}


Here, $\xi_{k}$ determines the importance of decreasing risk/variation compared to maximizing expected value. $\xi_{k}$ is called robust factor. Alternative $k$ will be preferred if $F_{k}^{*}>F_{i}^{*} \forall i, i \neq k$, i.e., alternative $k$ is considered to be the best if and only if

$$
E\left(F_{k}\right)-\xi_{k} \operatorname{var}\left(F_{k}\right) \geq E\left(F_{i}\right)-\xi_{k} \operatorname{var}\left(F_{i}\right) \quad \forall i, i \neq k .
$$

\subsection{Algorithmic approach}

Let $S=\left\{s_{1}, s_{2}, \ldots, s_{m}\right\}$ be the set of $m$ alternatives and $C=\left\{c_{1}, c_{2}, \ldots, c_{n}\right\}$ be the set of $n$ criteria. A decision maker expresses his/her opinion in linguistic terms, represented in a decision matrix as given below:

$$
D=\left(d_{i j}\right)_{m \times n}=\left[\begin{array}{cccc}
d_{11} & d_{12} & \cdots & d_{1 n} \\
d_{21} & d_{22} & \cdots & d_{2 n} \\
\vdots & \vdots & \ddots & \vdots \\
d_{m 1} & d_{m 2} & \cdots & d_{m n}
\end{array}\right]
$$

Here, $d_{i j}$ is a linguistic term to evaluate an alternative $s_{i}, 1 \leq i \leq m$ with respect to the criteria $c_{j}, 1 \leq j \leq n$. In this study, the linguistic terms are expressed in ITrFNs.

The steps in the algorithm for selecting the best alternative considering both expectation and variance are given below:

Step 1 FLQ is used to obtain the optimism degree of the decision maker.

Step 2 Ordered weight vector $w=\left\{w_{1}, w_{2}, \ldots, w_{n}\right\}^{T}$, $w_{j} \geq 0, \sum_{j=1}^{n} w_{j}=1$ is computed based on the optimism degree of decision maker as shown in (12). For an optimistic decision maker, the first few ordered weights have higher values, and for the pessimistic decision maker, the first few ordered weights have lower values. Step 3 For each alternative $s_{i}, 1 \leq i \leq m$, the decision maker's opinion for the attributes $c_{j}, 1 \leq j \leq n$ are ordered according to their Hamming distances from the positive ideal ITrFNs, as mentioned earlier in (3). Less distance signifies more important attribute, represented by $\mathrm{ITrFN}$.

Step 4 ITrFOWA operator, given in (14), is applied to compute the value of aggregated ITrFN for each alternative $s_{i}, 1 \leq i \leq m$, corresponding to their criteria $c_{j}, 1 \leq j \leq n$, using Step 2 and Step 3 .

Step 5 Score $S\left(s_{i}\right)$ and accuracy values $H\left(s_{i}\right) \forall i$ of the aggregated ITrFNs for the corresponding alternative $s_{i}, 1 \leq i \leq m$ are computed to rank the alternatives, which are already mentioned in Definition 2.3. It gives the optimal choice. In the following steps, we find robust decision, which may not be the same as the optimal choice.
Step 6 The expected value $E\left(F_{k}\right)$ of the aggregated ITrFN for each alternative $k$ and its variance, $\operatorname{var}\left(F_{k}\right), 1 \leq k \leq m$ are obtained, respectively, using Definitions 2.3 and 2.4 .

Step 7 Collective measure for each alternative $k, 1 \leq k \leq m$ is computed by combining its expectation and variance using (15).

Step 8 The range of $\xi_{k}$, which we call as an optimal interval of $\xi_{k}$ for each alternative $k, 1 \leq k \leq m$ is evaluated using (16), as given below:

$$
\begin{aligned}
& \xi_{k} \leq \min \left[\frac{E\left(F_{k}\right)-E\left(F_{i}\right)}{\operatorname{var}\left(F_{k}\right)-\operatorname{var}\left(F_{i}\right)}\right], \text { if }\left(\operatorname{var}\left(F_{k}\right)-\operatorname{var}\left(F_{i}\right)\right) \geq 0 \\
& \xi_{k} \geq \min \left[\frac{E\left(F_{k}\right)-E\left(F_{i}\right)}{\operatorname{var}\left(F_{k}\right)-\operatorname{var}\left(F_{i}\right)}\right], \text { if }\left(\operatorname{var}\left(F_{k}\right)-\operatorname{var}\left(F_{i}\right)\right) \leq 0
\end{aligned}
$$

$i=1,2, \ldots, k-1, k+1, \ldots, m$.

Step 9 The robust decision is to select $s_{k}$ if the optimal interval of $\xi_{k}$ is more than those of others.

Step 10 If $k$ has more than one value, then any one of the corresponding $s_{k}$ may be chosen.

\section{Application in site selection problem of nuclear power plant}

Selecting proper site for installing a nuclear power plant has become a crucial problem throughout the world, especially after the Fukushima (2011) and Chernobyl (1986) nuclear accidents. A number of factors are to be considered before installing a nuclear power plant at a particular location (Kirkwood 1982). Among those, seismological factor, availability of sufficient water, public health and safety, social and environmental policies are considered to be most important. A few researchers have studied on nuclear power plant site selection problem in the last decade. Kirkwood (1982) performed a series of screening steps to determine the site to be selected from the candidate sites for a plant as well as water resources. The author performed multi-objective decision analysis to evaluate the rank of the candidate sites and water resources. Ford et al. (1979) reviewed the alternative methodologies, those of which have been used for selecting the nuclear power plant site. They specified the objective of each methodology and developed attributes to measure the degree of usage of each objective. The authors also rated various methodologies based on various attributes.

To demonstrate the application of the proposed approach, we present a case study regarding the site selection for installing nuclear power plant. Nuclear power plant construction and operation in India is regulated by the Nuclear Power Corporation of India Ltd (NPCIL). Among 
the set of criteria developed by NPCIL, there are primarily seven criteria for selecting a proper site to install nuclear power plant, which are given below:

(a) Population density $\left(c_{1}\right)$ : Nuclear power plants should preferably be located in sparsely populated areas, those of which are at a considerable distance from the areas of large population. This is necessary to minimize community opposition and security risks and to reduce the complexity associated with emergency planning.

(b) Seismological factors $\left(c_{2}\right)$ : Seismological factors have a huge impact on the safety measures associated with nuclear power plants. Installing nuclear power plants in seismically unstable areas increases the costs of construction and operation.

(c) Geographical conditions $\left(c_{3}\right)$ : Geographical conditions directly affect the costs and risks associated with nuclear power plants. They influence environmental pollution, as well as the risk of natural disasters triggering a substantial release of radioactive material.

(d) Atmospheric characteristics $\left(c_{4}\right)$ : There are two main atmospheric considerations. The first one is whether extreme weather conditions could affect the safe and efficient operation of the nuclear power plant. Two examples of such events include cyclone and flood. The second consideration is how atmospheric conditions could affect the dispersion of radioactive material and other pollutants from routine releases and accidents. Relevant factors include prevailing winds, topographical factors that influence local climate (for example, hills and valleys), and risk of local fogging or icing due to water vapor discharge.

(e) Cooling water features $\left(c_{5}\right)$ : Sufficient water is needed for cooling purpose.

(f) Land use $\left(c_{6}\right)$ : Decision makers should consider the fact that installing a nuclear power plant puts industrial areas and freshwater resources at risk which may cause undesirable results.

(g) Economic conditions $\left(c_{7}\right)$ : The construction of nuclear power plants should be evaluated in terms of the cost of construction, the cost of building a power line and the cost of a cooling system. Minimizing these costs is necessary to reduce basic fixed costs, which makes an alternative more desirable.

This study has evaluated 13 sites with respect to these seven criteria mentioned above, considering a group of experts. The linguistic terms and their corresponding ITrFNs are provided in Table 2. The relationships among the linguistic terms and their corresponding ITrFNs have been considered in a consistent way motivated by the linguistic information given in Chen (2000) and Chen and Lee (2010). The final assessment of the experts given in the form of an assessment matrix is shown in Table 3, where assessments of decision makers/experts are given in linguistic terms.

A stepwise illustration of the solving procedure as per the proposed algorithm is presented below:

[Step 1] This study has used FLQ based on the number of criteria considered by the group of experts. If the group of experts consider more criteria for evaluating the alternatives, then they are considered to be more pessimistic. Here, we assume that experts select the quantifier 'many of them' from Table 1, for which the optimism degree is 0.333 . Here, the experts are considered as 'fairly pessimistic' as given in Table 1.

[Step 2] Considering optimism degree $\theta=0.333$ and number of criteria $n=7$, the order weights are evaluated using (12) as $[0.020,0.061,0.102,0.143,0.184,0.225$, $0.266]$.

[Step 3 and Step 4] Aggregated ITrFN of each site is computed using the ITrFOWA operator. The result is shown in Table 4.

Table 2 Linguistic terms and their corresponding ITrFNs

\begin{tabular}{ll}
\hline Linguistic term & Intuitionistic trapezoidal fuzzy number \\
\hline Very low (VL) & $([0,0.1,0.2,0.3] ; 0.6,0.3)$ \\
Low $(\mathrm{L})$ & $([0.05,0.15,0.25,0.35] ; 0.2,0.5)$ \\
Medium low (ML) & $([0.2,0.3,0.4,0.7] ; 0.7,0.1)$ \\
Medium (M) & $([0.35,0.45,0.5,0.65] ; 0.5,0.5)$ \\
Medium high (MH) & $([0.5,0.6,0.75,0.8] ; 0.3,0.6)$ \\
High $(\mathrm{H})$ & $([0.65,0.7,0.85,0.9] ; 0.4,0.5)$ \\
Very high $(\mathrm{VH})$ & $([0.8,0.9,1,1] ; 0.7,0.2)$ \\
\hline
\end{tabular}

Table 3 Assessment matrix expressed in linguistic terms

\begin{tabular}{llllllll}
\hline & $c_{1}$ & $c_{2}$ & $c_{3}$ & $c_{4}$ & $c_{5}$ & $c_{6}$ & $c_{7}$ \\
\hline$s_{1}$ & $\mathrm{VL}$ & $\mathrm{MH}$ & $\mathrm{L}$ & $\mathrm{ML}$ & $\mathrm{H}$ & $\mathrm{H}$ & $\mathrm{VH}$ \\
$s_{2}$ & $\mathrm{ML}$ & $\mathrm{MH}$ & $\mathrm{H}$ & $\mathrm{MH}$ & $\mathrm{ML}$ & $\mathrm{VH}$ & $\mathrm{H}$ \\
$s_{3}$ & $\mathrm{H}$ & $\mathrm{L}$ & $\mathrm{ML}$ & $\mathrm{VH}$ & $\mathrm{M}$ & $\mathrm{H}$ & $\mathrm{L}$ \\
$s_{4}$ & $\mathrm{VL}$ & $\mathrm{L}$ & $\mathrm{ML}$ & $\mathrm{H}$ & $\mathrm{MH}$ & $\mathrm{L}$ & $\mathrm{H}$ \\
$s_{5}$ & $\mathrm{VH}$ & $\mathrm{H}$ & $\mathrm{ML}$ & $\mathrm{ML}$ & $\mathrm{L}$ & $\mathrm{H}$ & $\mathrm{L}$ \\
$s_{6}$ & $\mathrm{H}$ & $\mathrm{VH}$ & $\mathrm{L}$ & $\mathrm{L}$ & $\mathrm{H}$ & $\mathrm{MH}$ & $\mathrm{VH}$ \\
$s_{7}$ & $\mathrm{~L}$ & $\mathrm{H}$ & $\mathrm{MH}$ & $\mathrm{ML}$ & $\mathrm{H}$ & $\mathrm{VH}$ & $\mathrm{M}$ \\
$s_{8}$ & $\mathrm{MH}$ & $\mathrm{VL}$ & $\mathrm{L}$ & $\mathrm{H}$ & $\mathrm{VL}$ & $\mathrm{H}$ & $\mathrm{L}$ \\
$s_{9}$ & $\mathrm{M}$ & $\mathrm{MH}$ & $\mathrm{ML}$ & $\mathrm{L}$ & $\mathrm{VH}$ & $\mathrm{H}$ & $\mathrm{ML}$ \\
$s_{10}$ & $\mathrm{VH}$ & $\mathrm{M}$ & $\mathrm{MH}$ & $\mathrm{ML}$ & $\mathrm{L}$ & $\mathrm{L}$ & $\mathrm{H}$ \\
$s_{11}$ & $\mathrm{VL}$ & $\mathrm{L}$ & $\mathrm{ML}$ & $\mathrm{H}$ & $\mathrm{L}$ & $\mathrm{ML}$ & $\mathrm{MH}$ \\
$s_{12}$ & $\mathrm{H}$ & $\mathrm{ML}$ & $\mathrm{L}$ & $\mathrm{VH}$ & $\mathrm{VL}$ & $\mathrm{L}$ & $\mathrm{M}$ \\
$s_{13}$ & $\mathrm{ML}$ & $\mathrm{MH}$ & $\mathrm{M}$ & $\mathrm{L}$ & $\mathrm{H}$ & $\mathrm{MH}$ & $\mathrm{ML}$ \\
\hline & & & & & & &
\end{tabular}


Table 4 Aggregated ITrFNs $([a, b, c, d] ; \mu, v)$ when $\theta=0.333$

\begin{tabular}{lllllll}
\hline & $a$ & $b$ & $c$ & $d$ & $\mu$ & $v$ \\
\hline$s_{1}$ & 0.253769 & 0.359901 & 0.464945 & 0.545585 & 0.471976 & 0.324806 \\
$s_{2}$ & 0.43258 & 0.557082 & 0.722392 & 0.787701 & 0.491153 & 0.317335 \\
$s_{3}$ & 0.239496 & 0.345627 & 0.458866 & 0.557876 & 0.403272 & 0.390032 \\
$s_{4}$ & 0.165044 & 0.271166 & 0.365995 & 0.4507 & 0.422856 & 0.353434 \\
$s_{5}$ & 0.21194 & 0.336442 & 0.477236 & 0.567061 & 0.337376 & 0.528727 \\
$s_{6}$ & 0.340456 & 0.428223 & 0.549642 & 0.620091 & 0.32822 & 0.479903 \\
$s_{7}$ & 0.340595 & 0.446727 & 0.571198 & 0.658975 & 0.420908 & 0.40634 \\
$s_{8}$ & 0.120126 & 0.21606 & 0.276189 & 0.367029 & 0.451189 & 0.396517 \\
$s_{9}$ & 0.294749 & 0.416162 & 0.545728 & 0.638599 & 0.460392 & 0.344888 \\
$s_{10}$ & 0.242622 & 0.349758 & 0.465044 & 0.557917 & 0.363752 & 0.430755 \\
$s_{11}$ & 0.137578 & 0.252855 & 0.350736 & 0.442582 & 0.446765 & 0.344721 \\
$s_{12}$ & 0.146672 & 0.253808 & 0.333344 & 0.435402 & 0.457609 & 0.363707 \\
$s_{13}$ & 0.313211 & 0.428488 & 0.559074 & 0.64478 & 0.399751 & 0.317335 \\
\hline
\end{tabular}

Table 5 Scores of alternatives

\begin{tabular}{llllll}
\hline & $\alpha=0.05$ & $\alpha=0.1$ & $\alpha=0.3$ & $\alpha=0.5$ & $\alpha=0.7$ \\
\hline$s_{1}$ & 0.01881 & 0.018719 & 0.034276 & 0.056584 & 0.133801 \\
$s_{2}$ & $\mathbf{0 . 0 6 9 1 5 8}$ & $\mathbf{0 . 0 5 6 5 6 7}$ & $\mathbf{0 . 0 6 3 7 5 3}$ & $\mathbf{0 . 1 0 2 1 5 8}$ & $\mathbf{0 . 1 7 3 3 5 8}$ \\
$s_{3}$ & 0.021024 & 0.021325 & 0.002686 & 0.040549 & 0.129416 \\
$s_{4}$ & 0.018602 & 0.014217 & 0.011627 & 0.012388 & 0.0148 \\
$s_{5}$ & 0.020942 & 0.022189 & 0.030806 & 0.01015 & 0.147386 \\
$s_{6}$ & 0.02108 & 0.023332 & 0.031178 & 0.019826 & 0.140463 \\
$s_{7}$ & 0.023501 & 0.030454 & 0.003727 & 0.045684 & 0.13547 \\
$s_{8}$ & 0.019475 & 0.018733 & 0.007059 & 0.007077 & 0.02194 \\
$s_{9}$ & 0.023501 & 0.030395 & 0.030524 & 0.090616 & 0.171914 \\
$s_{10}$ & 0.021079 & 0.023069 & 0.012623 & 0.032477 & 0.129666 \\
$s_{11}$ & 0.018601 & 0.013938 & 0.01664 & 0.044724 & 0.051852 \\
$s_{12}$ & 0.0186 & 0.013939 & 0.015013 & 0.049413 & 0.135905 \\
$s_{13}$ & 0.026792 & 0.034341 & 0.021695 & 0.058916 & 0.085057 \\
\hline
\end{tabular}

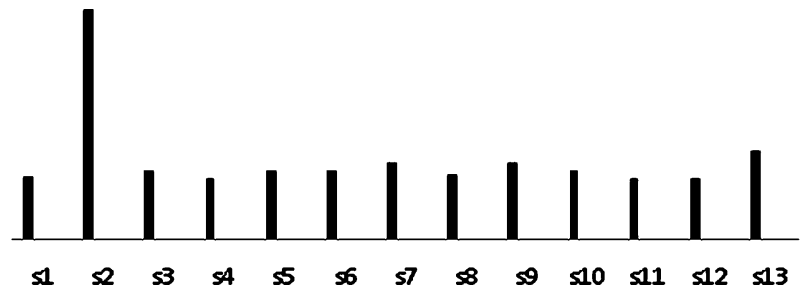

Fig. 1 Plot of score values when $\alpha=0.05$

[Step 5] The score values of various sites are evaluated based on a set of five different values of the quantifier $\alpha$, which are $0.05,0.1,0.3,0.5$, and 0.7 , given in Table 5. It is observed that site $s_{2}$ has the highest score value among all of the five values of $\alpha$, which are shown in bold face. Thus, site $s_{2}$ is the optimal choice of the decision makers. Five figures, Figs. 1, 2, 3, 4, and 5, are, respectively, the graphical representations of the score values of different

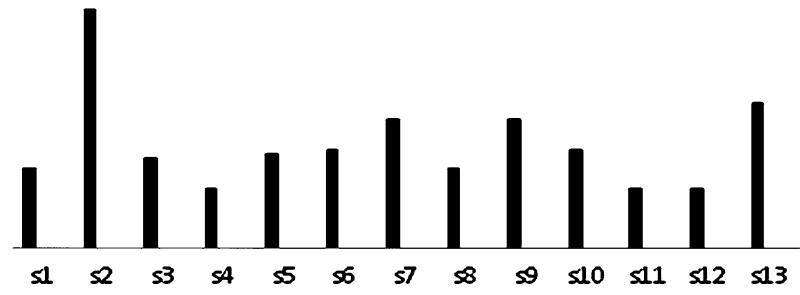

Fig. 2 Plot of score values of different sites when $\alpha=0.1$

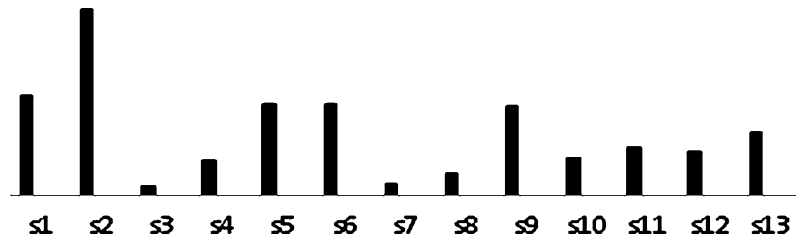

Fig. 3 Plot of score values of different sites when $\alpha=0.3$

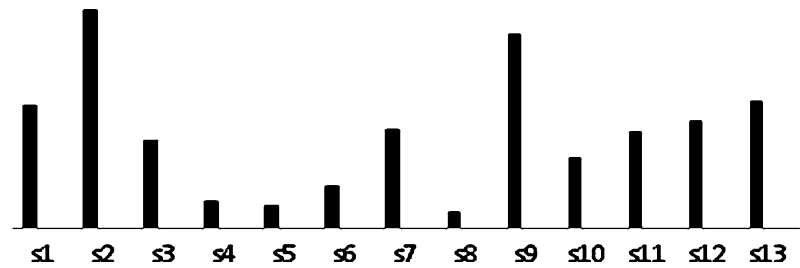

Fig. 4 Plot of score values of different sites when $\alpha=0.5$

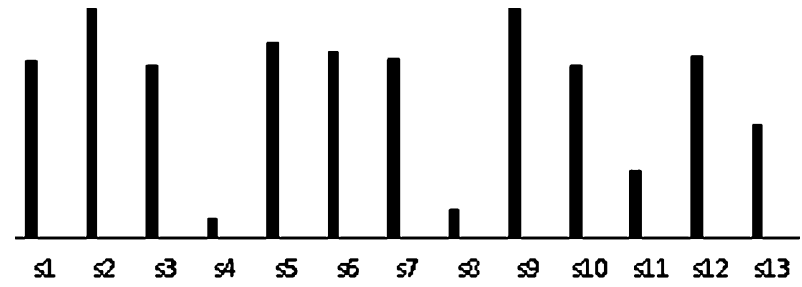

Fig. 5 Plot of score values of different sites when $\alpha=0.7$ 
Table 6 Expected value and variances of aggregated ITrFNs

\begin{tabular}{|c|c|c|c|c|c|c|c|c|c|c|}
\hline & \multicolumn{2}{|l|}{$\alpha=0.05$} & \multicolumn{2}{|l|}{$\alpha=0.1$} & \multicolumn{2}{|l|}{$\alpha=0.3$} & \multicolumn{2}{|l|}{$\alpha=0.5$} & \multicolumn{2}{|l|}{$\alpha=0.7$} \\
\hline & $E\left(F_{k}\right)$ & $\operatorname{Var}\left(F_{k}\right)$ & $E\left(F_{k}\right)$ & $\operatorname{Var}\left(F_{k}\right)$ & $E\left(F_{k}\right)$ & $\operatorname{Var}\left(F_{k}\right)$ & $E\left(F_{k}\right)$ & $\operatorname{Var}\left(F_{k}\right)$ & $E\left(F_{k}\right)$ & $\operatorname{Var}\left(F_{k}\right)$ \\
\hline$s_{1}$ & 0.106115 & 0.001666 & 0.146287 & 0.001658 & 0.40605 & 0.001609 & 0.558929 & 0.00132 & 0.72028 & 0.001044 \\
\hline$s_{2}$ & 0.662186 & 0.001205 & 0.653571 & 0.001319 & 0.624939 & 0.001893 & 0.678571 & 0.001569 & 0.773977 & 0.001104 \\
\hline$s_{3}$ & 0.200481 & 0.001669 & 0.21398 & 0.001721 & 0.400466 & 0.0018 & 0.548214 & 0.001453 & 0.712856 & 0.000659 \\
\hline$s_{4}$ & 0.105356 & 0.001667 & 0.127752 & 0.001659 & 0.313226 & 0.00165 & 0.455357 & 0.001214 & 0.602936 & 0.001004 \\
\hline$s_{5}$ & 0.20046 & 0.001671 & 0.213456 & 0.001778 & 0.39817 & 0.00216 & 0.546429 & 0.001707 & 0.711869 & 0.001228 \\
\hline$s_{6}$ & 0.200777 & 0.001666 & 0.223118 & 0.001634 & 0.484603 & 0.001132 & 0.6375 & 0.000934 & 0.771586 & 0.000851 \\
\hline$s_{7}$ & 0.224431 & 0.001641 & 0.307097 & 0.001598 & 0.504374 & 0.001682 & 0.614286 & 0.001387 & 0.745396 & 0.001077 \\
\hline$s_{8}$ & 0.100167 & 0.001667 & 0.105067 & 0.001666 & 0.244851 & 0.001484 & 0.401786 & 0.001162 & 0.574002 & 0.000795 \\
\hline$s_{9}$ & 0.224431 & 0.001641 & 0.306955 & 0.0016 & 0.47381 & 0.002113 & 0.571429 & 0.001955 & 0.712115 & 0.001464 \\
\hline$s_{10}$ & 0.20077 & 0.001666 & 0.221246 & 0.001648 & 0.403835 & 0.001758 & 0.532143 & 0.001612 & 0.694532 & 0.001271 \\
\hline$s_{11}$ & 0.105357 & 0.001667 & 0.127887 & 0.001665 & 0.295938 & 0.001945 & 0.4125 & 0.002033 & 0.557374 & 0.001503 \\
\hline$s_{12}$ & 0.105353 & 0.001677 & 0.126839 & 0.001671 & 0.292306 & 0.001857 & 0.451786 & 0.001684 & 0.651622 & 0.001304 \\
\hline$s_{13}$ & 0.22472 & 0.001639 & 0.314359 & 0.001547 & 0.486388 & 0.001879 & 0.533929 & 0.002033 & 0.616854 & 0.001519 \\
\hline
\end{tabular}

sites for five different values of $\alpha$, which are $0.05,0.1,0.3,0.5$, and 0.7 .

[Step 6] The expected value $E\left(F_{k}\right)$ and variance $\operatorname{var}\left(F_{k}\right)$ of the aggregated ITrFN for each site $s_{k}, k=1,2, \ldots, 13$ for five different values of the quantifier $\alpha$, are given in Table 6 . The result shows that site 2 , i.e., $s_{2}$ is the most preferred alternative as it has the highest expected value for all of the five values of $\alpha$, which are $0.05,0.1,0.3,0.5$, and 0.7. So site 2, i.e., $s_{2}$ can be considered as the optimal choice, which is the same as evaluated in the previous step, i.e., Step 5. We know that smaller variance represents safer decision. So, we compare the variance values and find that site 2, i.e., $s_{2}$ is best for $\alpha=0.05$ and $\alpha=0.1$. However, site 6, i.e., $s_{6}$ is best for $\alpha=0.3$ and $\alpha=0.5$. Site 3 , i.e., $s_{3}$ is best when $\alpha=0.7$. This shows the importance of combining variance with expectation, as illustrated below.

[Step 7 and Step 8] Optimal interval of $\xi_{k}$ as given in (16) for each site $s_{k}, k=1,2, \ldots, 13$ is determined using the collective measure of expected value and variance, which is shown in Table 7. Figure 6 illustrates the optimal interval of $\xi_{k}$ for each of the sites $s_{k}, k=1,2, \ldots, 13$ at $\alpha=0.1$. Similarly, Figs. 7, 8, 9, and 10 present the corresponding optimal intervals of all the 13 sites, respectively, for the remaining four values of $\alpha$, which are $0.05,0.3,0.5$, and 0.7 .

[Step 9] Robust decision is selected based on the larger optimal interval of $\xi_{k}, k=1,2, \ldots, 13$. Considering the uncertain behaviour of the decision parameters, one can decide that decision making in larger interval is more robust than that in smaller interval. Figure 6 shows site 4 , i.e., $s_{4}$ has to be selected for the robust decision when $\alpha=0.1$. Figures 7, 8, 9, and 10 present the respective robust decisions as selection of site 8 , site 13 , site 10 , and site 8 for $\alpha=0.05,0.3,0.5$, and 0.7. Different robust decisions are observed due to different values of $\alpha$, which are associated with different optimism degrees of the decision maker.

In this study, the opinions of decision makers are expressed using linguistic terms which have been transformed into ITrFNs. In real-life decision making, the information about any decision problem and its parameters is often incomplete and imprecise in nature. To handle these kinds of problems and due to the inherent fuzzy reasoning process of human beings, the decision makers always prefer linguistic terms to express their opinions. The linguistic terms are well represented by ITrFNs, since ITrFN uses trapezoidal function for both membership and non-membership degrees. The trapezoidal function supports different dimensions (Wang and Zhang 2009a) to express the uncertain information, which adds more flexibility to model the linguistic terms. Due to the presence of trapezoidal function, ITrFN is considered to be more powerful in expressing the linguistic information than intuitionistic fuzzy set. We have evaluated the optimal decision as well as the robust decision based on the optimism degree $\theta$ /parameter of quantifier $(\alpha)$ of the decision maker. A set of five values of the parameter of quantifier $\alpha$ has been used to analyze the problem. For each value of $\alpha$, i.e., $\alpha=0.05,0.1,0.3,0.5$, and $\alpha=0.7$, site 2 has been selected as the optimal decision among a set of 13 sites/ alternatives. But selection of site 2 has not been evaluated as the robust decision. The robust decision is found to be different for different values of $\alpha$. When $\alpha=0.05$, site 8 has to be selected for the robust decision. Similarly, when $\alpha=0.1,0.3,0.5$, and 0.7 , the respective robust decisions are selection of site 4 , site 13 , site 10 , and site 8 . This study 
Table 7 Optimal intervals of $\xi_{k}$ for each site $k$

\begin{tabular}{llllll}
\hline & $\alpha=0.05$ & $\alpha=0.1$ & $\alpha=0.3$ & $\alpha=0.5$ & $\alpha=0.7$ \\
\hline$s_{1}$ & {$[0,31455.33]$} & {$[1079.23,8574.5952]$} & {$[164.68,1346.90]$} & {$[202.06,861.455]$} & {$[229.74,894.95]$} \\
$s_{2}$ & {$[0.00,251.12]$} & {$[391.24,423.57]$} & {$[1011.23,1657.47]$} & {$[345.78,539.21]$} & {$[0,10.331]$} \\
$s_{3}$ & {$[0,995.05]$} & {$[0,1094.69]$} & {$[880.58,2413.69]$} & {$[991.87,1126.43]$} & {$[0,331.927]$} \\
$s_{4}$ & {$[0,47562.50]$} & {$[1397.245,18333.26]$} & {$[2263.99,5973.37]$} & {$[649.51,955.44]$} & {$[1134.96,2933.599]$} \\
$s_{5}$ & {$[0,990.83]$} & {$[0,959.81]$} & {$[0,1609.36]$} & {$[100.66,957.87]$} & {$[1.0423,500.87]$} \\
$s_{6}$ & {$[0,1000.89]$} & {$[0,241.724]$} & {$[0,184.41]$} & {$[0,64.63]$} & {$[0,0]$} \\
$s_{7}$ & {$[0,144.500]$} & {$[0,1243.29]$} & {$[0,571.40]$} & {$[51.24,352.56]$} & {$[138.01,1058.55]$} \\
$s_{8}$ & {$[0,50137.00]$} & {$[2851.57,13099.57]$} & {$[681.11,1310.72]$} & {$[1026.03,1032.84]$} & {$[1020.98,3617.57]$} \\
$s_{9}$ & {$[0,144.50]$} & {$[0,1235.62]$} & {$[0,686.95]$} & {$[0,277.32]$} & {$[0,171.84]$} \\
$s_{10}$ & {$[0,0]$} & {$[0,1728.66]$} & {$[1322.88,1637.80]$} & {$[150.31,3408.39]$} & {$[91.1036,475.72]$} \\
$s_{11}$ & {$[47562.00,95419.99]$} & {$[153.983,6001.90]$} & {$[1058.76,6326.94]$} & {$[0,0]$} & {$[3717.5,3967.718]$} \\
$s_{12}$ & {$[0,47564.00]$} & {$[1872.64,3786.59]$} & {$[1897.54,9239.8]$} & {$[1973.54,4099.418]$} & {$[378.08,1300.3]$} \\
$s_{13}$ & {$[0,1007.98]$} & {$[0,1486.68]$} & {$[91.29,9896.5]$} & {$[0,481.108]$} & {$[0,1732.018]$} \\
\hline
\end{tabular}

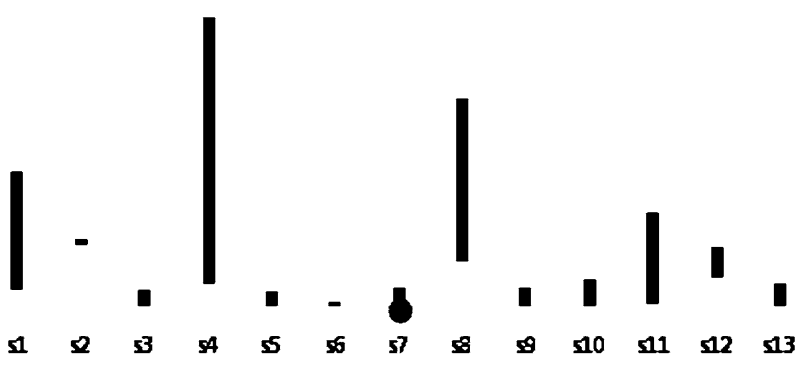

Fig. 6 Optimal intervals of 13 sites when $\alpha=0.1$

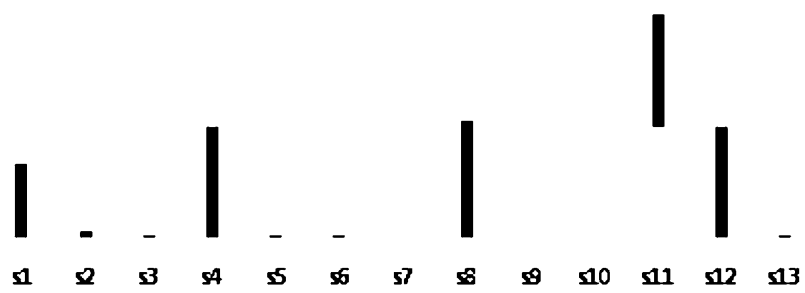

Fig. 7 Optimal intervals of 13 sites when $\alpha=0.05$

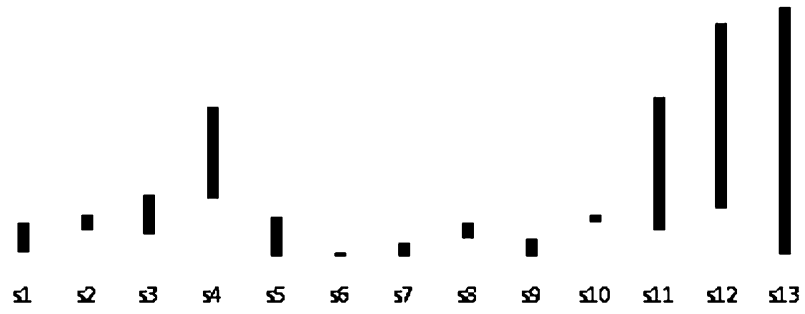

Fig. 8 Optimal intervals of 13 sites when $\alpha=0.3$

has combined expected value and variance to find out the robust decision, which tries to maximize the expected value and minimize the variance.

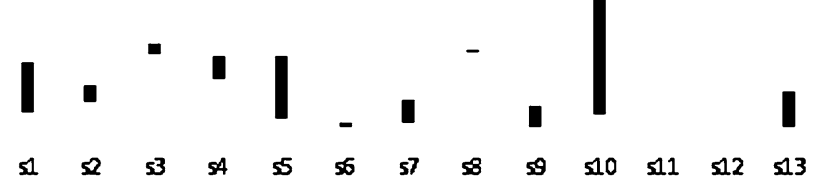

Fig. 9 Optimal intervals of 13 sites when $\alpha=0.5$

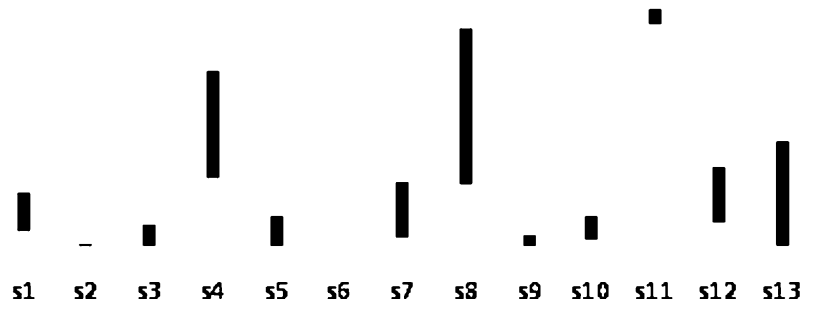

Fig. 10 Optimal intervals of 13 sites when $\alpha=0.7$

\section{Result discussion and comparison}

This section briefly discusses the outcome of the proposed approach and compares it with the relevant existing methods.

\subsection{Discussion on results}

This study initially computes the optimal choice of decision makers, which is shown in Table 5, using a set of five different values of the quantifier $\alpha, 0.05,0.1,0.3,0.5$, and 0.7. We find that site $s_{2}$ is selected as the optimal decision as it has the highest score value for all of the five values of 
$\alpha$. As we know that optimal decision is not always the robust decision, we proceed further to compute the robust decision using expected value and variance. In the process, we fist compute expected value of each site for the five different values of $\alpha(0.05,0.1,0.3,0.5,0.7)$ to measure the central tendency of each site which is considered as weighted average of the criteria values of that site. The expected values of 13 sites using five different values of $\alpha(0.05,0.1,0.3,0.5,0.7)$ are given in Table 6. From Table 6, we observe that site $s_{2}$ is again selected as optimal decision due to having highest expected value for all the five different values of the quantifier $\alpha$. When we consider variance, we observe that different sites are selected based on different values of the quantifier due to having less variance. It is known that higher expected value provides better decision and lower variation provides safer decision. Hence, a combination of expected value and variance will provide better as well as riskless decision. This leads us to combine expected value and variance to determine robust decision which is both better and less risky. Table 7 shows the necessary intervals for each of the 13 sites for the five different values of the quantifier $\alpha$. For each value of $\alpha$, site with largest interval is selected as robust decision.

\subsection{Comparative study}

The proposed decision making approach in this study is considered as an extension of the approach given in Zarghami et al. (2008). Zarghami et al. (2008) proposed fuzzy stochastic ordered weighted averaging (FSOWA) method to find robust decision in Sefidrud watershed problem, located in the northwestern region of Iran. Sefidrud is an important watershed in Iran, mainly used for water resources development, which has 13 projects on water resources. They initially performed a stochastic simulation on the input dataset and, then, investigated the robust project combining expected value and variance. Zarghami and Szidarovszky (2009) used a method, called as fuzzy stochastic ordered weighted averaging, to solve water resources management problem in the central Tisza River in Hungary. As an extension to the approach, given in Zarghami et al. (2008), and Zarghami and Szidarovszky (2009) used linguistic opinions of decision makers. Both authors, Zarghami et al. (2008) and Zarghami and Szidarovszky (2009), used stochastic fuzzy MCDM methods with a discrete set of alternatives, where they initially performed a stochastic simulation and, then, used FLQ to obtain the optimism degree of decision makers. Compared to the approaches, given in Zarghami et al. (2008) and Zarghami and Szidarovszky (2009), where the uncertainty is managed stochastically, we have used IFS, more concretely ITrFN, which is more close to the reasoning process of human being. ITrFNs are based on IFS and used to represent the opinions of decision makers. Instead of performing stochastic simulation, we have used intuitionistic trapezoidal fuzzy ordered weighted averaging (ITrFOWA) operator to determine the aggregated evaluation of each site corresponding to the various criteria. This aggregated evaluation, in turn, assists us to explore the optimal decision. Then, we proceed further to evaluate the robust decision using collective measure.

\section{Conclusions}

This paper has proposed a robust MCDM approach as an extension of the method of Zarghami et al. (2008) in the context of ITrFNs. Optimism degree of a decision maker is obtained from FLQ, which is represented by RIM quantifier. In this study, quantifier executes an important role to assign optimism degree to a decision maker. Less is the quantifier, more is the optimism degree. ITrFOWA operator has been used to aggregate ITrFNs for each of the alternatives. Optimal decision is evaluated by ranking the alternatives based on their score and accuracy values. However, an optimal decision may not be the robust one. To explore the robust decision, expected value and variances of each alternative are computed and, then, combined for a collective measure. Finally, robust decision is taken based on the optimal interval. More is the optimal interval, the decision is more robust. This study finds that the optimal decision is not necessarily the robust decision and vice versa. The proposed approach is demonstrated on a site selection problem of nuclear power plant. This study has shown the significance of robust decision in the changed scenario using a real-life example. The change in the scenario arises out of uncertainty. The uncertainty is represented by different parameters, like linguistic terms, quantifier, expectation, and variance. These parameters are used in the proposed method to tackle the uncertain behaviour of the power plant to be installed. If we consider robust decision instead of optimal one, the effect of nuclear hazards in case of an unexpected event due to changes in the parameters can be reduced. In future, the concept of robustness can be embedded to many decision making problems for making the decision robust. Since different types of criteria, such as benefit type and cost type, have been used in the decision process, to avoid deriving the wrong results, the decision information presented in the decision matrix may be normalized using the idea given in $\mathrm{Xu}$ and $\mathrm{Hu}$ (2010). Information granules can also be considered to design the robust system. 


\section{References}

Antonelli M, Ducange P, Lazzerini B, Marcelloni F (2016) Multiobjective evolutionary design of granular rule-based classifiers. Granul Comput 1:37-58

Apolloni B, Bassis S, Rota J, Galliani GL, Gioia M, Ferrari L (2016) A neurofuzzy algorithm for learning from complex granules. Granul Comput 1

Atanassov KT (1986) Intuitionistic fuzzy sets. Fuzzy Sets Syst 20:87-96

Atanassov KT (1999) Intuitionistic fuzzy sets. In: Intuitionistic Fuzzy sets, volume 35 of studies in fuzziness and soft computing. Physica-Verlag, Heidelberg, pp 1-137

Bargiela A, Pedrycz W (2012) Granular computing: an introduction, vol 717. Springer Science and Business Media, Berlin

Bui LT, Abbass HA, Barlow M, Bender A (2012) Robustness against the decision-maker's attitude to risk in problems with conflicting objectives. IEEE Trans Evolut Comput 6(1):1-19

Cabrerizo FJ, Herrera-Viedma E, Pedrycz W (2013) A method based on pso and granular computing of linguistic information to solve group decision making problems defined in heterogeneous contexts. Eur J Oper Res 230(3):624-633

Chen C-T (2000) Extensions of the topsis for group decision-making under fuzzy environment. Fuzzy Sets Syst 114(1):1-9

Chen S-M, Lee L-W (2010) Fuzzy multiple attributes group decisionmaking based on the interval type-2 topsis method. Expert Syst Appl 37(4):2790-2798

Ciucci D (2016) A triarchic theory of granular computing. Granul Comput 1:145-157

Deb K, Gupta H (2006) Introducing robustness in multi-objective optimization. Evolut Comput 14(4):463-494

Dey A, Pradhan R, Pal A, Pal T (2015) The fuzzy robust graph coloring problem. In: Proceedings of the 3rd international conference on frontiers of intelligent computing: theory and applications (FICTA) 2014, pp 805-813. Springer

Dubois D, Prade H (2016) Bridging gaps between several forms of granular computing. Granul Comput 1:115-126

Ford CK, Keeney RL, Kirkwood CW (1979) Evaluating methodologies: a procedure and application to nuclear power plant siting methodologies. Manag Sci 25(1):1-10

Gaspar-Cunha A, Covas JA (2008) Robustness in multi-objective optimization using evolutionary algorithms. Comput Optim Appl 39(1):75-96

Gupta SK, Rosenhead J (1968) Robustness in sequential investment decisions. Manag Sci 15(2):B-18

Hasuike T (2013) Robust shortest path problem based on a confidence interval in fuzzy bicriteria decision making. Inform Sci 221:520-533

Kirkwood CW (1982) A case history of nuclear power plant site selection. J Oper Res Soc 33:353-363

Kreinovich V (2016) Solving equations (and systems of equations) under uncertainty: how different practical problems lead to different mathematical and computational formulations. Granul Comput 1

Lempert RJ, Collins MT (2007) Managing the risk of uncertain threshold responses: comparison of robust, optimum, and precautionary approaches. Risk Anal 27(4):1009-1026

Lingras P, Haider F, Triff M (2016) Granular meta-clustering based on hierarchical, network, and temporal connections. Granul Comput 1:71-92

Liu H, Gegov A, Cocea M (2016) Rule-based systems: a granular computing perspective. Granul Comput 1

Livi L, Sadeghian A (2016) Granular computing, computational intelligence, and the analysis of non-geometric input spaces. Granul Comput 1:13-20
Loia V, D'Aniello G, Gaeta A, Orciuoli F (2016) Enforcing situation awareness with granular computing: a systematic overview and new perspectives. Granul Comput 1:127-143

Maciel L, Ballini R, Gomide F (2016) Evolving granular analytics for interval time series forecasting. Granul Comput 1

Malczewski J (2006) Ordered weighted averaging with fuzzy quantifiers: gis-based multicriteria evaluation for land-use suitability analysis. Int J Appl Earth Obs Geoinform 8(4):270-277

Mendel JM (2016) A comparison of three approaches for estimating (synthesizing) an interval type-2 fuzzy set model of a linguistic term for computing with words. Granul Comput 1:59-69

Mens MJP, Klijn F, de Bruijn KM, Beek EV (2011) The meaning of system robustness for flood risk management. Environ Sci Policy 14:1121-1131

Min F, Xu J (2016) Semi-greedy heuristics for feature selection with test cost constraints. Granul Comput 1

Nag K, Pal T, Pal NR (2014) Robust consensus: A new measure for multicriteria robust group decision making problems using evolutionary approach. In: Artificial intelligence and soft computing. Springer, Berlin, pp 384-394

Pedrycz W, Chen SM (2015) Granular computing and decisionmaking: interactive and iterative approaches. Springer, Berlin

Peters G, Weber R (2016) DCC: a framework for dynamic granular clustering. Granul Comput 1:1-11

Rosenhead J, Elton M, Gupta SK (1972) Robustness and optimality as criteria for strategic decisions. Oper Res Quarter 23:413-431

Simon HA (1959) Theories of decision making in economics and behavioral science. Am Econ Rev 49:253-283

Skowron A, Jankowski A, Dutta A (2016) Interactive granular computing. Granul Comput 1:95-113

Song M, Wang Y (2016) A study of granular computing in the agenda of growth of artificial neural networks. Granul Comput 1

Srinivasan T, Rethinaraj TG (2013) Fukushima and thereafter: reassessment of risks of nuclear power. Energy Policy $52: 726-736$

Wang J, Zhang Z (2009a) Aggregation operators on intuitionistic trapezoidal fuzzy number and its application to multi-criteria decision making problems. J Syst Eng Electron 20(2):321-326

Wang J, Zhang Z (2009b) Multi-criteria decision making method with incomplete certain information based on intuitionistic fuzzy number. Control Decis 24(2):226-230

Wang Z, Tian F (2010) A note of the expected value and variance of fuzzy variables. Int J Nonlinear Sci 9(4):486-492

Wei G (2010) Some arithmetic aggregation operators with intuitionistic trapezoidal fuzzy numbers and their application to group decision making. J Comput 5(3):345-351

Weinberg AM (1986) Nuclear power advocate reflects on chernobyl. Bull At Sci (United States) 43(1)

Wilke G, Portmann E (2016) Granular computing as a basis of human-data interaction: a cognitive cities use case. Granul Comput 1

$\mathrm{Xu} \mathrm{Z} \mathrm{(2007)} \mathrm{Intuitionistic} \mathrm{fuzzy} \mathrm{aggregation} \mathrm{operators.} \mathrm{IEEE} \mathrm{Trans}$ Fuzzy Syst 15(6):1179-1187

Xu Z, Hu H (2010) Projection models for intuitionistic fuzzy multiple attribute decision making. Int $\mathrm{J}$ Inform Technol Decis Mak 9(02):267-280

Xu Z, Wang H (2016) Managing multi-granularity linguistic information in qualitative group decision making: an overview. Granul Comput 1:21-35

Xu Z, Yager RR (2006) Some geometric aggregation operators based on intuitionistic fuzzy sets. Int J Gen Syst 35(4):417-433

Xue Y, Li D, Shan W, Wang C (2007) Multi-objective robust optimization using probabilistic indices. In: Third international conference on natural computation, 2007. ICNC 2007, vol 4, pp 466-470. IEEE 
Yager RR (1988) On ordered weighted averaging aggregation operators in multicriteria decisionmaking. IEEE Trans Syst Man Cybern 18(1):183-190

Yager RR (1996) Quantifier guided aggregation using owa operators. Int J Intell Syst 11(1):49-73

Yager RR (2002) On the cardinality index and attitudinal character of fuzzy measures. Int J Gen Syst 31(3):303-329

Yanez J, Ramirez J (2003) The robust coloring problem. Eur J Oper Res 148(3):546-558

Yao Y (2016) A triarchic theory of granular computing. Granul Comput 1:145-157

Ye J (2012) Multicriteria group decision making method using the distances-based similarity measures between intuitionistic trapezoidal fuzzy numbers. Int J Gen Syst 41(7):729-739
Zadeh LA (1975a) The concept of a linguistic variable and its application to approximate reasoning-III. Inform Sci 9(1):43-80

Zadeh LA (1975b) The concept of a linguistic variable and its application to approximate reasoning-I. Inform Sci 8(3):199-249

Zarghami M, Szidarovszky F (2009) Stochastic-fuzzy multi criteria decision making for robust water resources management. Stoch Environ Res Risk Assess 23(3):329-339

Zarghami M, Szidarovszky F, Ardakanian R (2008) A fuzzystochastic owa model for robust multi-criteria decision making. Fuzzy Optim Decis Mak 7(1):1-15 